Ludwig A. Th. Greif, Alexander Mittelstädt, Stefan T. Jagsch, Andrei Schliwa

\title{
Twofold gain enhancement by elongation of QDs in polarization preserving QD-SOAs
}

Journal article | Accepted manuscript (Postprint)

This version is available at https://doi.org/10.14279/depositonce-10475

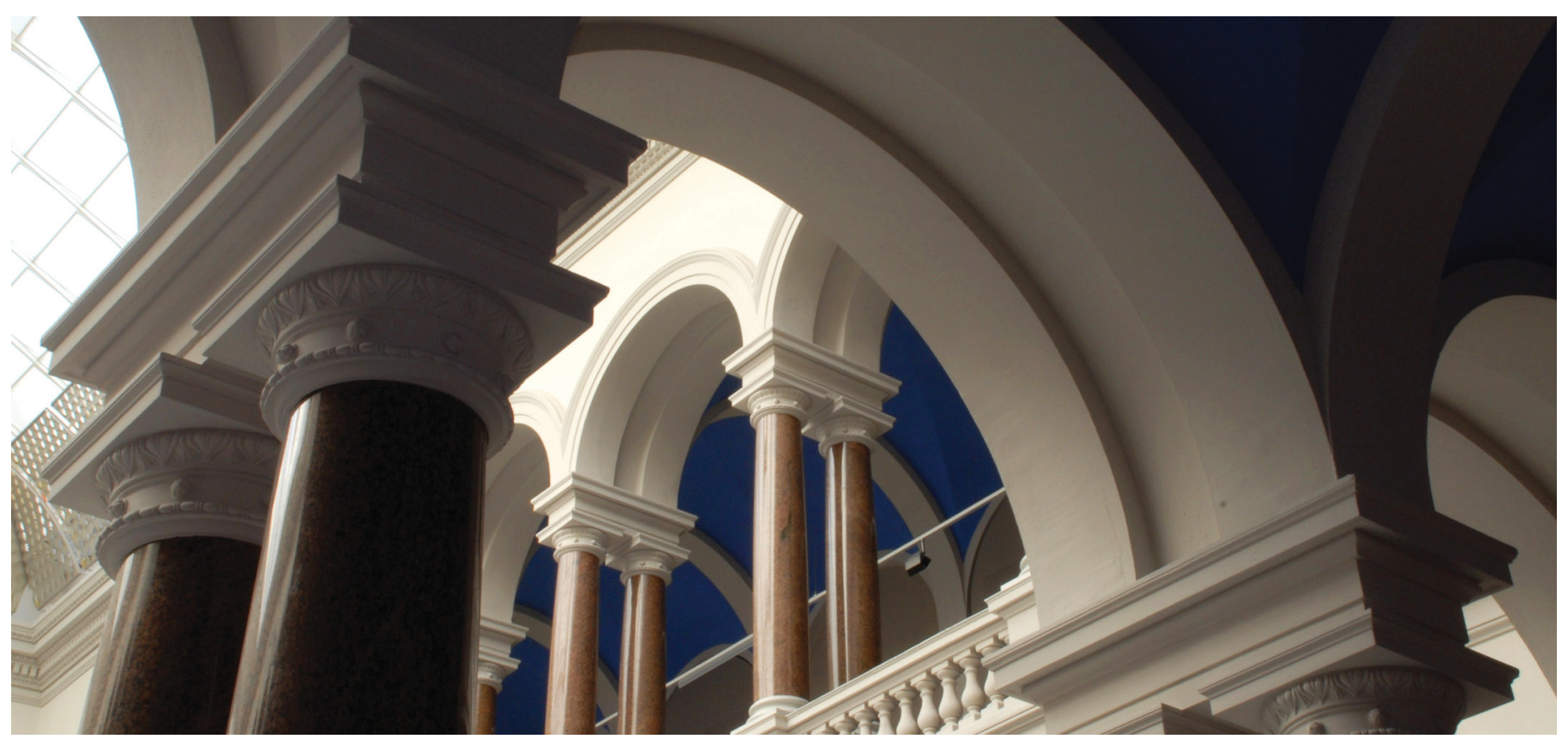

This is the Accepted Manuscript version of an article accepted for publication in Semiconductor Science and Technology. IOP Publishing Ltd is not responsible for any errors or omissions in this version of the manuscript or any version derived from it. The Version of Record is available online at https://doi.org/10.1088/1361-6641/ab1c06.

Greif, L. A. T., Mittelstädt, A., Jagsch, S. T., \& Schliwa, A. (2019). Twofold gain enhancement by elongation of QDs in polarization preserving QD-SOAs. Semiconductor Science and Technology, 34(7), 075003. https://doi.org/10.1088/1361-6641/ab1c06

\section{Terms of Use}

This work is licensed under a CC BY-NC-ND 3.0 License (Creative 
ACCEPTED MANUSCRIPT

\section{Twofold gain enhancement by elongation of QDs in polarization preserving QD-SOAs}

To cite this article before publication: Ludwig A. Th. Greif et al 2019 Semicond. Sci. Technol. in press https://doi.org/10.1088/1361-6641/ab1c06

\section{Manuscript version: Accepted Manuscript}

Accepted Manuscript is "the version of the article accepted for publication including all changes made as a result of the peer review process, and which may also include the addition to the article by IOP Publishing of a header, an article ID, a cover sheet and/or an 'Accepted

Manuscript' watermark, but excluding any other editing, typesetting or other changes made by IOP Publishing and/or its licensors"

This Accepted Manuscript is @ 2019 IOP Publishing Ltd.

During the embargo period (the 12 month period from the publication of the Version of Record of this article), the Accepted Manuscript is fully protected by copyright and cannot be reused or reposted elsewhere.

As the Version of Record of this article is going to be / has been published on a subscription basis, this Accepted Manuscript is available for reuse under a CC BY-NC-ND 3.0 licence after the 12 month embargo period.

After the embargo period, everyone is permitted to use copy and redistribute this article for non-commercial purposes only, provided that they adhere to all the terms of the licence https://creativecommons.org/licences/by-nc-nd/3.0

Although reasonable endeavours have been taken to obtain all necessary permissions from third parties to include their copyrighted content within this article, their full citation and copyright line may not be present in this Accepted Manuscript version. Before using any content from this article, please refer to the Version of Record on IOPscience once published for full citation and copyright details, as permissions will likely be required. All third party content is fully copyright protected, unless specifically stated otherwise in the figure caption in the Version of Record.

View the article online for updates and enhancements. 


\title{
Twofold Gain Enhancement by Elongation of QDs in Polarization Preserving QD-SOAs
}

\author{
LA Th Greif,* A Mittelstädt, S T Jagsch, and A Schliwa \\ Technische Universität Berlin, Institut für Festkörperphysik, Hardenbergstraße 36, 10623 \\ Berlin, Germany \\ E-mail: lath@physik.tu-berlin.de
}

\section{Abstract}

The impact of quantum dot (QD) elongation on key parameters of QD-based semiconductor optical amplifiers (SOAs) is investigated using a combination of 8-band $\boldsymbol{k} \cdot \boldsymbol{p}$-theory including strain and piezoelectricity up to second order and a rate equation model describing the population of QD ground, excited and wetting layer states. By considering columnar QDs of selected aspect ratios, we show that chip gain and saturation gain can be enhanced by up to $+3.6 \mathrm{~dB}$ via an increased elongation of the individual QDs while retaining polarization preserving amplification and gain recovery times below $700 \mathrm{fs}$. Our results enable the optimization of polarization preserving QD-SOA devices which combine ultrafast gain recovery with high gain and low power consumption.

\section{Keywords}

self-assembled quantum dots, semiconductor optical amplifier, $\boldsymbol{k} \cdot \boldsymbol{p}$-theory, gain enhancement

\section{Introduction}

Since the first pioneering works predicted most desirable electronic and dynamic properties for quantum dots (QDs), ${ }^{1,2}$ they have been in the focus of research for use in semiconductor optical amplifiers (SOAs). Ultrafast gain recovery and pronounced nonlinear effects ${ }^{3}$ substantiate the high potential of QD-SOAs in the fields of high frequency signal amplification, all-optical data processing and wavelength conversion using four-wave-mixing $(\mathrm{FWM})^{4-7}$. Deyices with/modulation frequencies exceeding $200 \mathrm{GHz}^{8}$ and a $120 \mathrm{~nm}$ broadband gain with saturation output powers above $20 \mathrm{~dB}$ facilitate wavelength division multiplexing ${ }^{9,10}$ and high efficiency symmetric FWM. ${ }^{11}$ Furthermore, several experimental and theoretical works have shown that, using closely stacked QDs, polarization preserving amplification can be realized. ${ }^{12-18} \mathrm{~A}$ remaining drawback is the lower chip gain of QD-SOAs when compared to quantum well-based systems, requiring longer or multiple gain devisions to achieve comparable amplification. $^{9}$

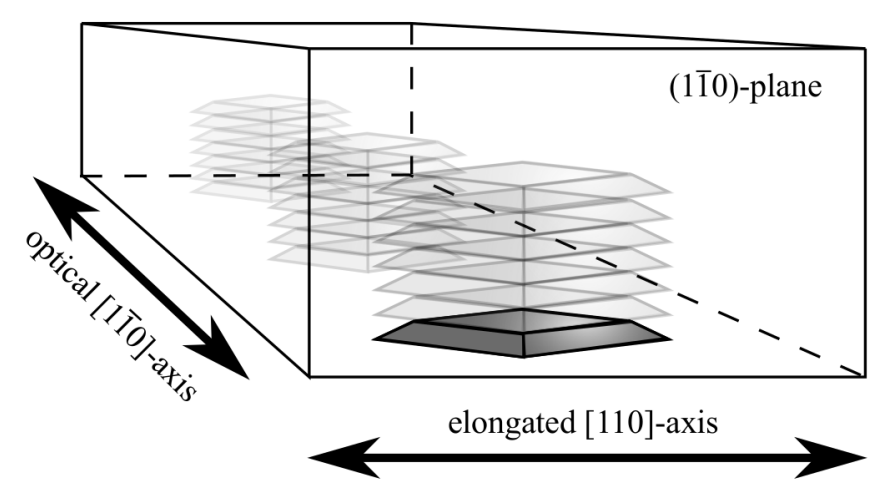

Figure 1: Scheme of the active region of a QDSOA with closely stacked QDs, showing the axis of elongation with respect to the optical axis.

In this paper, we study an approach to in- 
crease the chip gain of a QD-SOA while simultaneously preserving the input signal polarization. The gain enhancement is realized using $c$-plane QDs that are elongated perpendicular to the optical [1ㅣㅣ-axis of the device (figure 1). Several growth studies have demonstrated that such elongated QDs can be realized with molecular beam epitaxy (MBE) as well as metalorganic vapor phase epitaxy (MOVPE) via adjusting the growth temperature or including annealing steps. ${ }^{19-21}$ However, the formation of a uniform elongation of all QDs within one stack is still challenging. ${ }^{22}$

Due to the asymmetric confinement in the (001)-plane the QD ground state transition dipole moment is rotated into the (110)-plane, optimizing the alignment with the electric field of the optical signal. Thereby, the polarization of the amplified signal is preserved in the (110)plane. The results are not limited to this unique configuration of optical axis and QD-axis but can be generalized as long as the axis of elongation is orientated perpendicular to the optical axis of the QD-SOA.

Anisotropic transition dipole moments caused by anisotropic nanostructures were already used in InAs/InP quantum dash lasers and SOAs. ${ }^{23-25}$ Quantum dashes represent an intermediate structure between QDs and quantum wires with at least one dimension significantly exceeding the exciton Bohr radius and thus exhibit only two-dimensional quantum confinement. ${ }^{26}$ Due to the bigger volume compared to QDs, the threshold current is usually slightly higher than in QD based structures ${ }^{27}$ and although polarization insensitive amplification seems achievable ${ }^{28}$ to our best knowledge it has not been demonstrated so far.

The paper is organized as follows. After introducing the device structure, a brief paragraph discusses the method for calculating the spatially resolved radiation intensities of a stack of QDs. QD stacks with a vanishing degree of polarization (DOP) in the (110)-plane are then calculated for five different horizontal aspect ratios $A R_{\mathrm{h}}=d_{[110]} / d_{[1 \overline{1} 0]}$ (elongations), where $d_{i}$ is the length of the diagonal $[i]$ in the basis plane. Subsequently, the results are used to determine the impact of the QD elongation on the stimulated emission rate as well as other key properties of the QD-SOA, such as chip gain, saturation gain and gain recovery time $(G R T)$.

\section{Structure Details}

Typical QD-SOA designs use the tilted ridge waveguide geometry, ${ }^{5,29,30}$ where the cross section provides refractive/index $n$ guiding along the vertical and gain guiding in lateral direction (figure 2). Following Jayavel et al., ${ }^{31}$ we use $\mathrm{Al}_{0.5} \mathrm{Ga}_{0.5}$ As for the cladding $(n=3.1)$ and GaAs $(n=3.3)$ as matrix material in order to realize the index guiding.

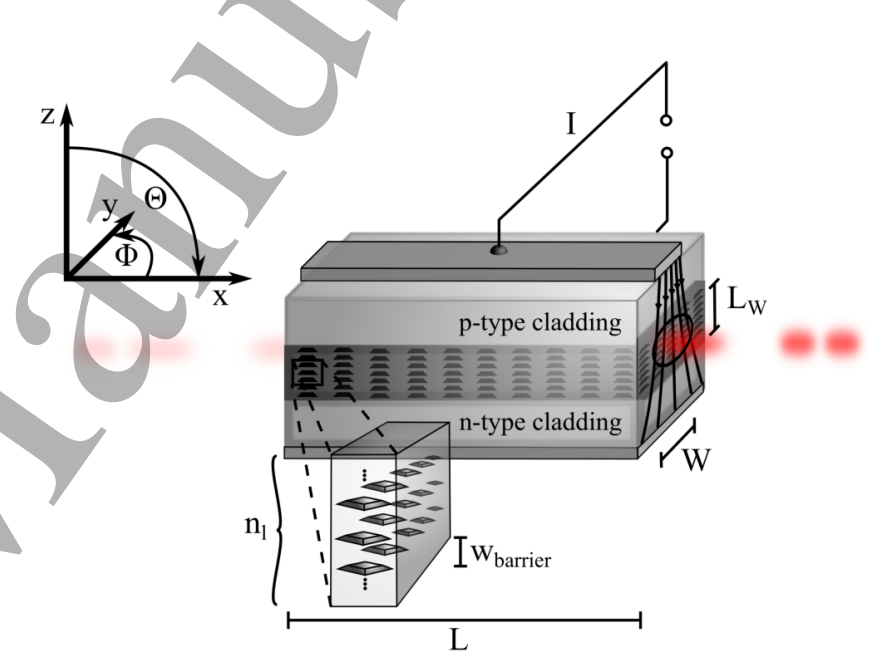

Figure 2: Schematic QD-SOA with pump current $I$, a resulting current injection region of width $W$, height of the active region $L_{\mathrm{W}}$, cavity length $L$ and $n_{1}$ layers of quantum dots, each separated by a barrier of width $w_{\text {barrier }}$.

Figure 2 shows the active region composed of seven layers of closely stacked QDs aligned along the $c$-axis. The QDs are placed on a twomonolayer (ML)-thick wetting layer (included in the QD height). The barrier width $w_{\text {barrier }}$ is set to zero ML, as experimental findings suggest that columnar QDs stay in contact with each other along the growth direction. ${ }^{12,14,15}$ The individual QDs are modeled as truncated InAspyramids with a side-wall angle of $30^{\circ}$ and dimensions given in table 1 .

As shown in $[13,32,33]$, elongation affects the polarization properties of single and stacked QDs. Thus, when changing the QD elongation, 
Table 1: Geometric specifications of the individual QDs.

\begin{tabular}{lll}
\hline$A R_{\mathrm{h}}$ & QD height & $d_{[110]}[\mathrm{nm}]$ \\
\hline 1.0 & 1.13 & 12.39 \\
1.5 & 1.13 & 11.19 \\
2.0 & 1.41 & 12.39 \\
2.5 & 1.70 & 12.39 \\
3.0 & 1.13 & 7.59 \\
\hline
\end{tabular}

adjustments in the geometry of the individual QDs are necessary to preserve polarization insensitivity of the stacks. Differences in the QD volume are of no particular significance as only the spatial radiation pattern enters the subsequent simulations of the QD-SOA dynamics as discussed in section 3.2.

Device and operational parameters are adapted from $[34,35]$ and listed in table 2 .

\section{Method of Calculation}

In order to connect macroscopic device properties like electric current and photon density with the elongation of the zero dimensional QDs, we use the rate equation model described in [34-37] accompanied by numerically obtained QD emission characteristics. In a first step, the radiation pattern of the QD stacks with different elongations are calculated using an 8-band $\boldsymbol{k} \cdot \boldsymbol{p}$ - model including strain and strain-induced piezoelectricity. ${ }^{38,39}$ Subsequently, the radiation pattern is used to determine the ratio of photons spontaneously emitted into the waveguide, known as $\beta$-factor

$$
\beta=\frac{\# \text { spon. emitted along the waveguide }}{\# \text { all spon. emitted }} .
$$

This figure of merit enters the rate equation model and allows us to qualitatively study the impact of the QD elongation on the device properties via its influence on the electron and hole wavefunctions.

\subsection{Beta-Factor and Spontaneous Emission Rate}

In order to obtain the rate of photons emitted by the QDs within the waveguide structure, we use Fermi's golden rule

$$
\Gamma_{\mathrm{i} \rightarrow \mathrm{f}}(\boldsymbol{\epsilon}) \propto|\boldsymbol{\epsilon} \cdot\langle f|\boldsymbol{\nabla}| i\rangle|^{2} .
$$

The polarization dependent transition rate $\Gamma_{\mathrm{i} \rightarrow \mathrm{f}}(\boldsymbol{\epsilon})$ can be derived with the help of the 8band $\boldsymbol{k} \cdot \boldsymbol{p}$ electron and hole wavefunctions $|f\rangle$ and $|i\rangle$, respectively. Following [40], $\Gamma_{\mathrm{i} \rightarrow \mathrm{f}}(\boldsymbol{\epsilon})$ is transformed into a function of the propagation direction $\boldsymbol{k}$. Only photons whose propagation direction $\boldsymbol{k}\left(\phi_{\mathrm{k}}, \theta_{\mathrm{k}}\right)$ fulfills the following equations $^{41}$ are confined within the waveguide

$$
\begin{aligned}
& \theta_{\mathrm{k}} \leq \arccos \left(\frac{\hat{n}_{2}}{\hat{n}_{1}}\right)=\theta_{\mathrm{lim}}, \\
& \phi_{\mathrm{k}} \leq \arctan \left(\frac{W}{2(L-x)}\right)=\phi_{\lim } .
\end{aligned}
$$

Here, $\hat{n}_{1}, \hat{n}_{2}$ are the refractive index of the matrix/and cladding material, respectively, $W$ is the width of the injection region, $L$ is the SOA length and $x$ is the position in the SOA along the optical axis. We eliminate the spatial dependence of (4) by averaging the limit angle $\phi_{\text {lim }}$ over the active region

$$
\bar{\phi}_{\lim }=\frac{1}{L} \int_{0}^{L} d x \phi_{\lim }(x) .
$$

With the help of the calculated limit angles, the $\beta$-factor can be derived as the ratio of the sum of all photons emitted within the limit angles to the total sum of emitted photons.

\subsection{Rate Equation Model}

The rate equation model is adapted from $[34,35,37]$ with corrections suggested by Li and $\mathrm{Li}$ in [36]. The model describes the temporal evolution of the charge carrier densities within the wetting layer $N_{\mathrm{W}}$, the QD excited state $N_{2}=\tilde{N}_{\mathrm{Q}} n_{2}$ and ground state $N_{1}=\tilde{N}_{\mathrm{Q}} n_{1}$. Here, $n_{2}$ and $n_{1}$ are the occupation probabilities of excited state and ground state, respectively. Occupation probabilities and carrier 
densities are connected to the QD volume density $\tilde{N}_{\mathrm{Q}}=N_{\mathrm{Q}} / L_{\mathrm{W}}$ via the $\mathrm{QD}$ surface density $N_{\mathrm{Q}}$.

Lifetimes for the capture $\tau_{\mathrm{W} \rightarrow 2}$ and intradot relaxation $\tau_{2 \rightarrow 1}$ are calculated considering phonon-mediated and Auger processes ${ }^{42}$

$$
\tau_{\mathrm{i}}=\frac{1}{A_{i}+C_{i} N_{\mathrm{W}}}, \quad i=(W \rightarrow 2),(2 \rightarrow 1),
$$

where $A_{\mathrm{i}}$ and $C_{\mathrm{i}}$ are the coefficients of phononand Auger-assisted interaction, respectively. The lifetimes are connected to the excitation $\tau_{1 \rightarrow 2}$ and escape process $\tau_{2 \rightarrow \mathrm{W}}$ via a quasi-Fermi equilibrium condition ${ }^{42}$ at $T=300 \mathrm{~K}$,

$$
\begin{aligned}
\tau_{2 \rightarrow \mathrm{W}} & =\tau_{\mathrm{W} \rightarrow 2} \frac{2 \rho_{2} N_{\mathrm{Q}} h^{2}}{4 \pi m_{\mathrm{e}}^{*} k_{\mathrm{B}} T} \exp \left(\frac{E_{\mathrm{W}}-E_{2}}{k_{\mathrm{B}} T}\right), \\
\tau_{1 \rightarrow 2} & =\tau_{2 \rightarrow 1} \frac{\rho_{1}}{\rho_{2}} \exp \left(\frac{E_{2}-E_{1}}{k_{\mathrm{B}} T}\right) .
\end{aligned}
$$

Here, $\rho_{1}$ and $\rho_{2}$ denote the ground and excited state degeneracy without spin, $h$ is the Planck constant, $m_{\mathrm{e}}^{*}$ is the effective electron mass, $k_{\mathrm{B}}$ is the Boltzmann constant and $E_{\mathrm{W}}$, $E_{2}, E_{1}$ are the energies of the wetting layer, excited and ground state. For the considered wetting layer carrier concentrations $N_{\mathrm{W}}$ the relaxation and excitation processes are mainly phonon- $\left(N_{\mathrm{W}}<1 \mathrm{e} 17 \mathrm{~cm}^{-3}\right)$ or Auger-assisted $\left(N_{\mathrm{W}}>1 \mathrm{e} 17 \mathrm{~cm}^{-3}\right)$, while the escape and capture processes are dominated by phonon creation/annihilation, showing nearly no $N_{\mathrm{W}}$ dependence (figure 3). Considering the internal losses $\alpha_{\text {int }}$, the optical power within the SOA can be calculated with the propagation equation $^{43}$

$$
P(z)=P_{\text {in }} \exp \left(\left(g-\alpha_{\text {int }}\right) z\right) .
$$

The dependence of the modal gain $g$ on the ground state occupation probability $n_{1}$ can be written as $g=g_{\max }\left(2 n_{1}-1\right) .{ }^{43}$ In various studies $g_{\max }$ is treated as a constant parameter depending only on structural properties of the specific device. ${ }^{34-36}$ As we want to compare QDSOAs with differently elongated QDs, a description of $g_{\max }$ as function of the elongation, or ef-

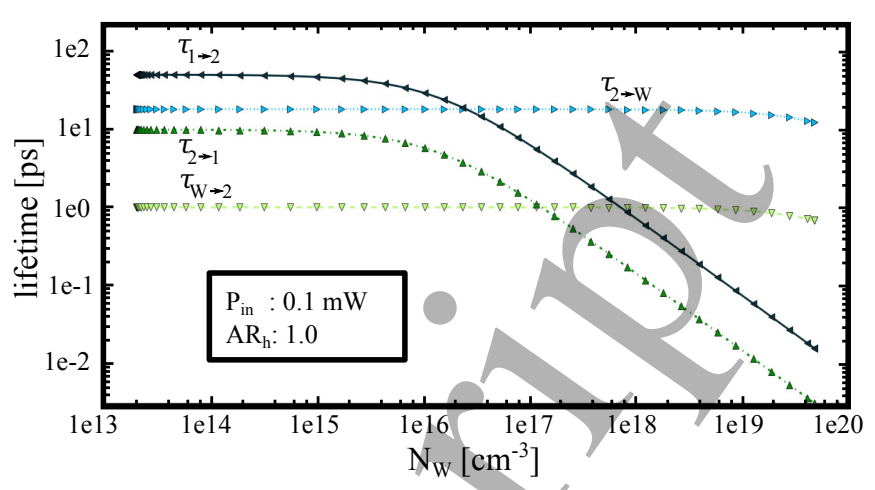

Figure 3: Lifetimes of capture $(\mathrm{W} \rightarrow 2)$, relaxation $(2 \rightarrow 1)$, escape $(2 \rightarrow W)$ and excitation $(1 \rightarrow 2)$ processes for different wetting layer carrier concentrations $N_{\mathrm{W}}$.

fectively as function of the $\beta$-value, is necessary. Following the microscopic derivation of $g_{\max }$ in $[43,44]$ a linear dependence on $\beta$ is revealed. Defining $g_{\max }^{0}$ for a QD-SOA with unelongated QDs the rate equation model is given as

$$
\begin{aligned}
\frac{\partial N_{\mathrm{W}}}{\partial t}= & \frac{J}{e L_{\mathrm{W}}}+\frac{N_{2}}{\tau_{2 \rightarrow \mathrm{W}}}- \\
& \frac{N_{\mathrm{W}}\left(1-n_{2}\right)}{\tau_{\mathrm{W} \rightarrow 2}}-\frac{N_{\mathrm{W}}}{\tau_{\mathrm{WR}}}, \\
\frac{\partial N_{2}}{\partial t}= & \frac{N_{\mathrm{W}}\left(1-n_{2}\right)}{\tau_{\mathrm{W} \rightarrow 2}}+\frac{N_{1}\left(1-n_{2}\right)}{\tau_{1 \rightarrow 2}}- \\
& \frac{N_{2}}{\tau_{2 \rightarrow \mathrm{W}}}-\frac{N_{2}\left(1-n_{1}\right)}{\tau_{2 \rightarrow 1}}, \\
\frac{\partial N_{1}}{\partial t}= & \frac{N_{2}\left(1-n_{1}\right)}{\tau_{2} \rightarrow 1}-\frac{N_{1}\left(1-n_{2}\right)}{\tau_{1 \rightarrow 2}}- \\
& \frac{N_{1} n_{1}}{\tau_{1 \mathrm{R}}}-\frac{g_{\max }^{0} \beta\left(2 n_{1}-1\right) \bar{P}}{\beta_{0} \sigma h \nu}
\end{aligned}
$$

with the injection current density $J=I /(L W)$, the elementary charge $e$, the thickness of the active layer $L_{\mathrm{W}}$, the radiative lifetime in the wetting layer and $\mathrm{GS}, \tau_{\mathrm{WR}}, \tau_{1 \mathrm{R}}$, the spatial average of the optical power within the SOA $\bar{P}$, the total cross section of the QDs $\sigma=V_{\mathrm{QDs}} / L$, with the total volume of the QDs $V_{\mathrm{QDs}} \cdot{ }^{36}$

Equations (10)-(12) are solved using the fourth-order Runge-Kutta method and an incremental time step of $1 \mathrm{e}-4 \mathrm{ps}$ except for 
Table 2: Parameters used in the rate equation model and the lifetime calculation.

\begin{tabular}{lll}
\hline parameter & physical meaning & value \\
\hline$I$ & injection current & $2 \mathrm{e}-7 \mathrm{~A}-20 \mathrm{~A}$ \\
$L_{\mathrm{W}}$ & thickness of effective active layer & $200 \mathrm{~nm}$ \\
$L_{\mathrm{WL}}$ & thickness of wetting layers & $3.5 \mathrm{~nm}$ \\
$L$ & length of the SOA & $2 \mathrm{~mm}$ \\
$W$ & width of current injection region & $10 \mu \mathrm{m}$ \\
$N_{\mathrm{Q}}$ & surface QD density & $5 \mathrm{e} 10 \mathrm{~cm}^{2}$ \\
$A_{\mathrm{W} \rightarrow 2}$ & phonon assisted trans. coeff. & $1 \mathrm{e} 12 \mathrm{~s}^{-1}$ \\
$C_{\mathrm{W} \rightarrow 2}$ & Auger assisted trans. coeff. & $1 \mathrm{e}-14 \mathrm{~m}^{3} \mathrm{~s}^{-1}$ \\
$A_{2 \rightarrow 1}$ & phonon assisted trans. coeff. & $1 \mathrm{e} 11 \mathrm{~s}^{-1}$ \\
$C_{2 \rightarrow 1}$ & Auger assisted trans. coeff. & $7 \mathrm{e}-12 \mathrm{~m}^{3} \mathrm{~s}^{-1}$ \\
$\rho_{2}$ & ES degeneracy (w/o spin) & 2 \\
$\rho_{1}$ & GS degeneracy (w/o spin) & 1 \\
$m_{\mathrm{e}}^{*}$ & effective electron mass & $0.0223 \mathrm{~m}_{\mathrm{e}}$ \\
$E_{\mathrm{W}}-E_{2}$ & energy splitting W $\rightarrow 2$ & $80 \mathrm{meV}$ \\
$E_{2}-E_{1}$ & energy splitting $2 \rightarrow 1$ & $60 \mathrm{meV}$ \\
$\tau_{\mathrm{WR}}$ & spon. radiative lifetime in wetting layer & $10 \mathrm{ps}$ \\
$\tau_{1 \mathrm{R}}$ & spon. radiative lifetime in GS & $400 \mathrm{ps}$ \\
$\alpha_{\text {int }}$ & internal losses & $3 \mathrm{~cm}^{-1}$ \\
$g_{\mathrm{max}}^{0}$ & modal gain of a QD-SOA with unelongated QDs & $11.5 \mathrm{~cm}^{-1}$ \\
\hline
\end{tabular}

the gain recovery calculations which were performed with a time step of $1 \mathrm{e}-5 \mathrm{ps}$.

Finally, the power of the amplified signal $P_{\text {out }}$ is calculated

$$
P_{\text {out }}=P_{\text {in }} \exp \left(\left(g-\alpha_{\text {int }}\right) L\right),
$$

which defines the chip gain $G$ in $\mathrm{dB}$

$$
G=10 \log _{10}\left(P_{\text {out }} / P_{\text {in }}\right) .
$$

The absolute energies of all states shift with different elongations as shown in [39]. But considering the more important energy spacings, the bandgap as well as the conduction interband spacing of the ground and first excited state are relatively constant. However, the interband spacing for higher states as well as the energy difference to the wetting layer reservoir is much more complex. This work constitutes a proof of concept and thus we refrain from simulations of the extended wetting layer and also from an explicit treatment of several higher excited states which would introduce a large number of unknown parameters.
In our opinion, such a simplified electronic picture is most suitable to first prove the impact of the elongation onto the basic device dynamics. Thus, except for the $\beta$-factor which is directly calculated using the QD stack wavefunction, all parameters are treated as constant for all considered QDs (table 2) with values similar to those used in $[34,35]$.

\section{Results}

Using single-particle states of the 8-band $\boldsymbol{k} \cdot \boldsymbol{p}$-simulations, the DOP and the $\beta$-factor can be determined for each elongation of the stacked QDs. Table 3 shows how the $\beta$-factor of the QD stack increases with the elongation of the individual QDs, while maintaining near-isotropic polarization through the stack aspect ratio. The increase of the $\beta$-factor is most pronounced for slightly elongated structures between $A R_{\mathrm{h}}=1.0$ and 1.5 and saturates rapidly for higher aspect ratios. In combination with the monotonous increase of $\beta$ only a small number of elongations has to be considered to 
Table 3: $D O P$ and $\beta$-factor of differently elongated QD stacks.

\begin{tabular}{ccc}
\hline$A R_{\mathrm{h}}$ & DOP & $\beta[\%]$ \\
\hline 1.0 & 0.08 & 1.10 \\
1.5 & -0.03 & 1.30 \\
2.0 & -0.01 & 1.41 \\
2.5 & -0.03 & 1.48 \\
3.0 & 0.10 & 1.50 \\
\hline
\end{tabular}

map the qualitative impact of $A R_{\mathrm{h}}$ onto the device properties.

According to (3) and (4), $\beta$ also depends on the waveguide dimensions as well as the material compositions. Therefore, the $\beta$-values may vary for identical QD stacks in a waveguide with different dimensions or refractive index.

\subsection{Gain Enhancement}

Having shown that we can efficiently tune the $\beta$ factor using elongated QDs, we now investigate the influence of the elongation on the chip gain, the GRT and the saturation gain.

In figure 4 the QD occupation probabilities $n_{2}$ and $n_{1}$ as well as the wetting layer carrier concentration $N_{\mathrm{W}}$ are shown as functions of $J$ and elongation for a constant optical input signal of $0.1 \mathrm{~mW}$. For $J<0.1 \mathrm{~A} / \mathrm{cm}^{2}$ both QD states $\mathrm{ex}^{-}$ hibit constant occupations. Here, a small $N_{\mathrm{W}}$ below $1 \mathrm{e} 14 \mathrm{~cm}^{-3}$ leads to small capture rates $N_{\mathrm{W}}\left(1-n_{2}\right) / \tau_{\mathrm{W} \rightarrow 2}$ and a long intradot relaxation time $\tau_{2 \rightarrow 1}$ (figure 3 ). Thus, the ground state is mainly populated due to the constant input signal, resembling an optically pumped two-level system with a steady state occupation of 0.5 . In combination with a negligible excitation process $(1 \rightarrow 2) n_{1}$ significantly exceeds $n_{2}$ $\left(n_{2} \ll n_{1}\right)$.

If $N_{\mathrm{W}}$ is increased via an increased injection current, the QD refilling takes place more efficiently and thus $n_{2}$ increases to unity $\left(n_{2}>n_{1}\right)$. This point represents the formation of an intradot relaxation bottleneck where $n_{1}$ is limited by the finite intradot relaxation time. A larger $N_{\mathrm{W}}$ also causes smaller intradot relaxation and excitation times, changing the dominating mechanism from phonon- to Auger-assisted (figure 3). Both processes exhibit different $N_{\mathrm{W}}$ dependencies which leads to the formation of a shoulder in $n_{1}$ around $J=10 \mathrm{~A} / \mathrm{cm}^{2}$. However, the relaxation always exceeds the excitation processes, resulting in a net growth of $n_{1}$ with increasing $J$. Ultimately, both states are completely occupied (upper limit of the relaxation bottleneck) for current densities above $3 \mathrm{e} 4 \mathrm{~A} / \mathrm{cm}^{2}$.

With QD elongation the transition dipole moment is increasingly aligned with the electric field of the propagating optical signal in the (110)-plane. Thus, the rate of stimulated emission increases, reducing $n_{1}$. Elongation-induced differences in the occupation probability can solely be observed in the relaxation bottleneck regime for the following reasons.

Firstly, for $J<0.1 \mathrm{~A} / \mathrm{cm}^{2}$, the ground state is mainly occupied due to the resonant optical signal resembling an optically pumped two-level system. This means that absorption and emission processes exactly compensate each other. Consequently, elongation-induced changes in the rate of stimulated emission do not have any impact on the QD state occupations.

Secondly, for $J>3 \mathrm{e} 4 \mathrm{~A} / \mathrm{cm}^{2}$, the optical losses are overcompensated by high capture rates and fast intradot relaxation processes. Thus, also in this regime no elongation dependence of $n_{1}$ can be found.

In contrast to the QD occupation, the impact of elongation on the chip gain $G$ is not limited to the relaxation bottleneck regime but can be observed as long as $G>0$ (figure $4 \mathrm{~b}$ ). Due to the amplified signal-to-QD coupling, the rate of stimulated emission is increased even for identical ground state occupations, leading to an elongation-induced gain enhancement.

Figure 5 a shows the QD occupation as a function of the signal output power. The QD occupation probabilities $n_{2}$ and $n_{1}$ are calculated for a realistic operating current density of $J=$ $5 \mathrm{e} 2 \mathrm{~A} / \mathrm{cm}^{2}$ and different QD elongations. ${ }^{10}$ For a wide range of output powers $\left(P_{\text {out }}<0.1 \mathrm{~mW}\right)$ the occupation is constant for all elongations meaning that the complete inversion of the QD ground states can be preserved $\left(n_{1}=1\right)$ due to the fast refilling dynamic on a femto- to picosecond timescale (figure 3). 

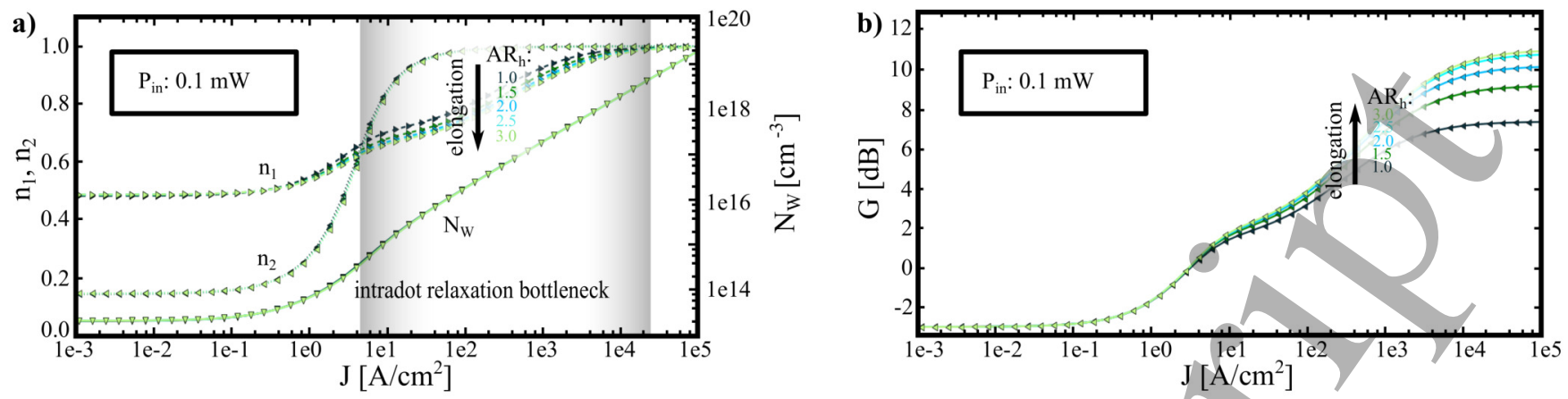

Figure 4: a) Wetting layer carrier concentration $N_{\mathrm{W}}$ (solid), steady state occupation probabilities of the QD ground state $n_{1}$ (dashed) and excited state $n_{2}$ (dotted) as well as $\mathbf{b}$ ) chip gain as function of injection current density for QD-SOAs with differently elongated QDs at a signal input power of $0.1 \mathrm{~mW}$.
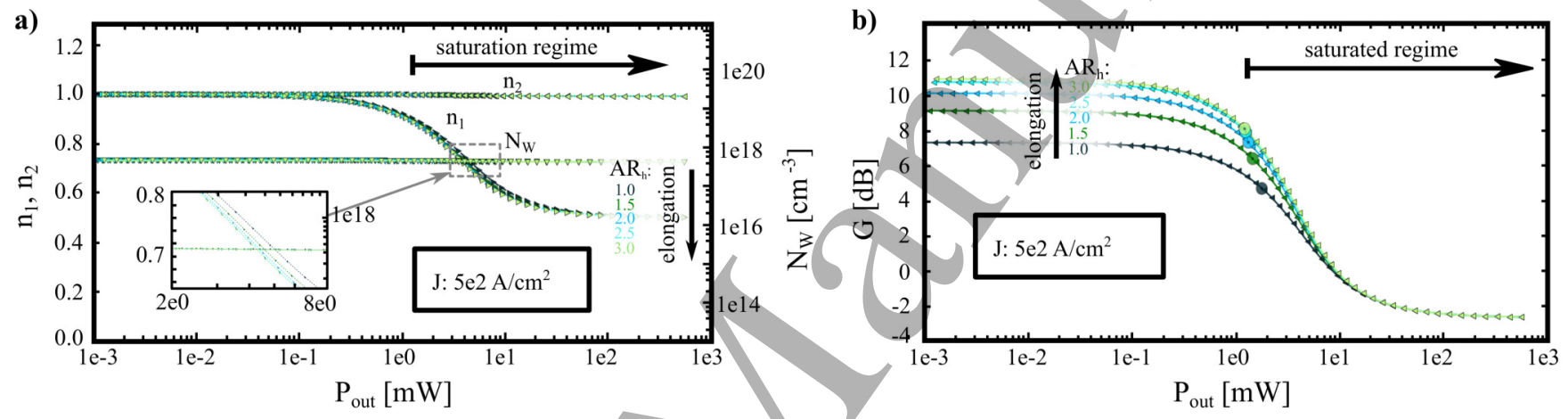

Figure 5: a) Wetting layer carrier concentration $N_{\mathrm{W}}$ (solid), steady state occupation probabilities of the QD ground state $n_{1}$ (dashed) and excited state $n_{2}$ (dotted) as well as $\mathbf{b}$ ) chip gain as function of output power for QD-SOAs with differently elongated QDs at an injection current density of $5 \mathrm{e} 2 \mathrm{~A} / \mathrm{cm}^{2}$. Circles mark the saturation output power and the associated saturation gain.

For output powers in the range of $10 \mathrm{~mW}$ the ground state occupation collapses and is driven into transparency $\left(n_{1}=0.5\right)$ representing a saturated gain medium. Like in an optically pumped two-level system (figure $4, J<$ $0.1 \mathrm{~A} / \mathrm{cm}^{2}$ ), optical gain and loss processes are compensating each other and, again, $n_{1}$ does not depend on the QD elongation. Solely for the transition regime between $0.1 \mathrm{~mW}<P_{\text {out }}<$ $10 \mathrm{~mW}$ the elongation-dependent increase in signal-to-QD coupling results in slightly reduced occupation probabilities (figure $5 \mathrm{a}$ inset).

For output powers below $0.1 \mathrm{~mW}$, the chip gain as a function of the output power (figure $5 \mathrm{~b}$ ) provides an elongation-induced gain enhancement of up to $+3.6 \mathrm{~dB}$. These results are in good agreement with an investigation

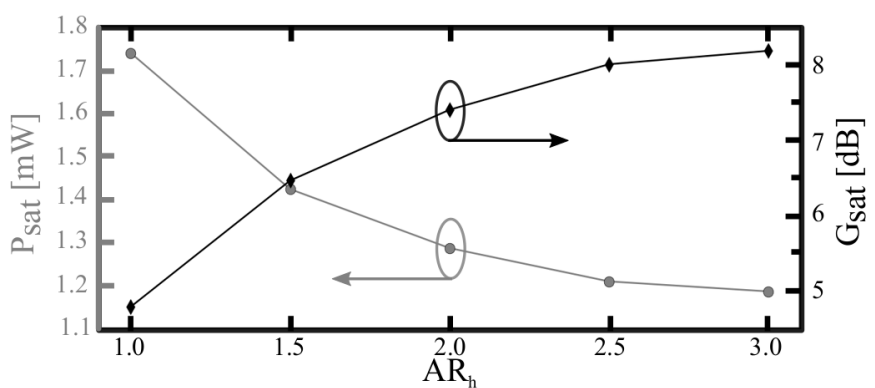

Figure 6: Saturation power (grey circles) and saturation gain (black diamonds) for QD-SOAs with differently elongated QDs.

on quantum dash SOAs whose gain can be increased by $+3.0-4.1 \mathrm{~dB}$ if the optical axis is chosen perpendicular to the quantum dashes, instead of being aligned. ${ }^{24}$

The enhancement is reduced with higher out- 
put powers and completely disappears in the saturated regime. In addition to the gain enhancement, the stronger signal-to-QD coupling also shifts the transition to the saturated regime towards lower output powers.

As a figure of merit for this transition the saturation power $P_{\text {sat }}$ is calculated, defined as the power for which $G$ is halfway between the low and high power limit ${ }^{45}$

$$
\begin{aligned}
P=P_{\text {sat }} \Leftrightarrow G(P) & =\left(G_{\text {low }}+\left|G_{\text {high }}\right|\right) / 2 \\
& =G_{\text {sat }} .
\end{aligned}
$$

The saturation power and the saturation gain are depicted in figure $5 \mathrm{~b}$ and figure 6 , respectively, showing that the saturation gain can be enhanced by $+3.4 \mathrm{~dB}$ even though the saturation power decreases with QD elongation. Strikingly, $70 \%(+2.6 \mathrm{~dB})$ of the maximum gain enhancement can be achieved already for $A R_{\mathrm{h}}=2$.

\subsection{Reduction of Gain Recovery Time}

The gain recovery time is analyzed by simulating a monochromatic pump-probe experiment (figure 7). ${ }^{34,35}$ To this end, a continuous wave probe signal of variable optical input power monitors the gain collapse and subsequent recovery following a pump pulse. A Gaussian pump pulse with a FWHM of $150 \mathrm{fs}$ and a peak power of $0.01 \mathrm{~mW}$ is inserted after the electrically driven system reaches a steady state. The time evolution of the out-coupled probe signal then determines the (90:10)\% GRT as a function of output power and elongation.

The GRT is determined by the competition between the intradot relaxation rate $\left(\Gamma_{2 \rightarrow 1}\right)$, excitation rate $\left(\Gamma_{1 \rightarrow 2}\right)$, rate of stimulated $\left(\Gamma_{\text {stim }}\right)$

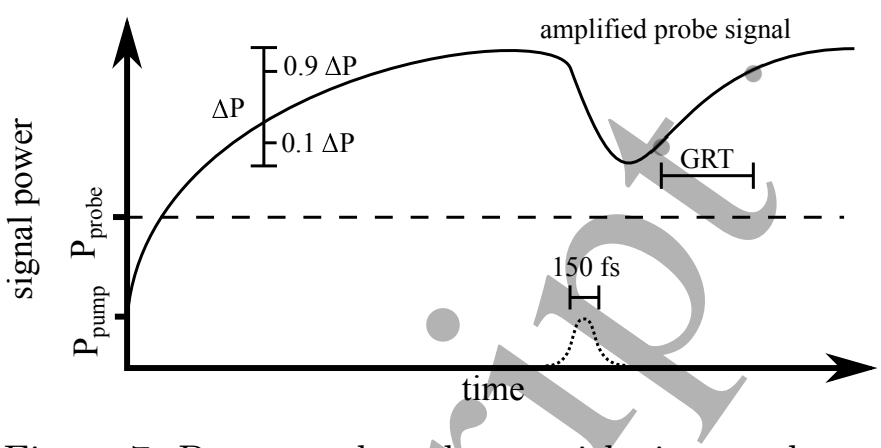

Figure 7: Pump-probe scheme with time evolution of input (pump - dotted; probe - dashed) and amplified probe signal (solid). The amplified probe signal declines by $\Delta P$ due to the pump insertion. The time to recover from $10 \%$ to $90 \%$ of $\Delta P$ defines the $G R T$.

and spontaneous emission $\left(\Gamma_{\text {spon }}\right)$

$$
\begin{aligned}
\Gamma_{2 \rightarrow 1} & =\frac{N_{2}\left(1-n_{1}\right)}{\tau_{2 \rightarrow 1}}, \\
\Gamma_{1 \rightarrow 2} & =\frac{N_{1}\left(1-n_{2}\right)}{\tau_{1 \rightarrow 2}}, \\
\Gamma_{\text {stim }} & =g_{\text {max }}\left(2 n_{1}-1\right) \frac{\bar{P}}{\sigma h \nu}, \\
\Gamma_{\text {spon }} & =\frac{N_{1} n_{1}}{\tau_{1 \mathrm{R}}}
\end{aligned}
$$

Except for the stimulated emission rate, all rates depend on a specific lifetime and QD state occupation probability. While the spontaneous emission lifetime is treated as a constant, the relaxation and excitation lifetimes depend on the wetting layer carrier density (6)-(8).

The lifetimes show no variation with respect to the elongation and only a small step-like increase of up to $11 \%$ near the saturation power (plots available in supporting informations)

$$
\begin{aligned}
\tau_{\mathrm{W} \rightarrow 2} & =18.25 \mathrm{ps} \text { to } 18.26 \mathrm{ps}, \\
\tau_{2 \rightarrow \mathrm{W}} & =1.00 \mathrm{ps}, \\
\tau_{1 \rightarrow 2} & =1.58 \mathrm{ps} \text { to } 1.72 \mathrm{ps} \\
\tau_{2 \rightarrow 1} & =0.31 \mathrm{ps} \text { to } 0.34 \mathrm{ps} .
\end{aligned}
$$

In contrast, the ground state occupation probability $n_{1}$ exhibits an elongation dependence (figure 5 a inset) becoming smaller for increasing elongation and intermediate output powers $0.1 \mathrm{~mW}<P_{\text {out }}<10 \mathrm{~mW}$. For this reason, the in-scattering rate $\Gamma_{2 \rightarrow 1}$ increases while 
the out-scattering rates $\Gamma_{1 \rightarrow 2}$ and $\Gamma_{\text {spon }}$ decrease. Considering the chip gain $G$ for differently elongated QDs as a function of the output power (figure $5 \mathrm{~b}$ ), we infer that the rate of stimulated emission is increased with elongation as long as $P_{\text {out }}$ is lower than $10 \mathrm{~mW}$. Additionally, $\Gamma_{\text {stim }}$ is also proportional to $\bar{P}$ and thus negligible for very low optical powers $\left(P_{\text {out }}<0.1 \mathrm{~mW}\right)$.

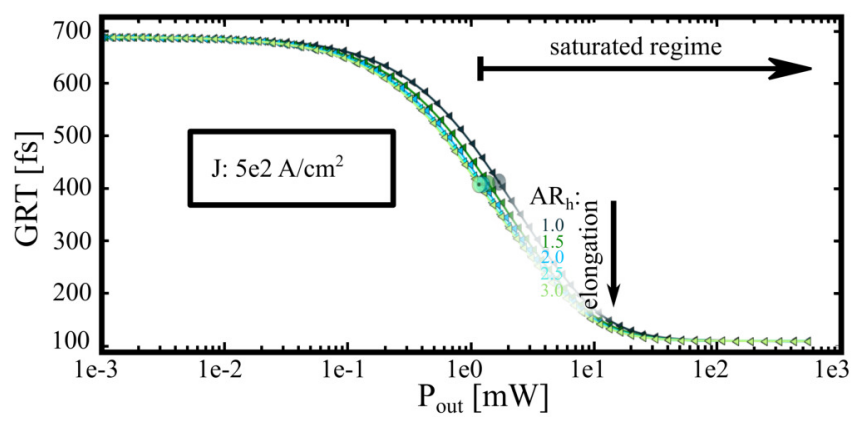

Figure 8: GRT as function of the output power for QD-SOAs with differently elongated QDs. Circles mark the saturation output power and the associated saturation $G R T$.

Consequently, the GRT as function of optical power and elongation (figure 8) exhibits neither power nor elongation dependence for $P_{\text {out }}$ lower than $0.1 \mathrm{~mW}$ or higher than $10 \mathrm{~mW}$. For low optical powers the GRT is significantly longer than the excited state to ground state relaxation time $\tau_{2 \rightarrow 1}$ due to out-scattering processes. This situation is reversed for high optical powers, since high photon absorption rates within the quasi-two-level regime (discussed in section A) result in a GRT much smaller than $\tau_{2 \rightarrow 1}$.

In the intermediate regime, the $G R T$ can be reduced by 60 fs by increasing $A R_{\mathrm{h}}$ from 1 to 3 . This reduction is caused by the net increase of the sum of all rates, as the increased losses $\Gamma_{\text {stim }}$ are overcompensated by the elongation-induced increase in in-scattering $\Gamma_{2 \rightarrow 1}$ and the reduced losses $\Gamma_{1 \rightarrow 2}$ and $\Gamma_{\text {spon }}$.

Analogous to the saturation gain the saturation $G R T$ can be determined $G R T_{\text {sat }}=$ $G R T\left(P_{\text {sat }}\right)$ (figure 9). In contrast to the $G R T$ for a given output power in the intermediate regime, $G R T_{\text {sat }}$ is relatively insensitive to the elongation.

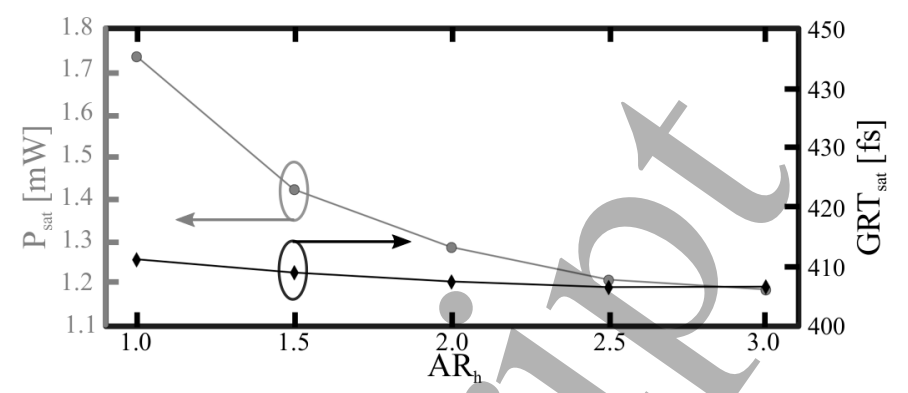

Figure 9: Saturation power (grey circles) and saturation GRT (black diamonds) for QDSOAs with differently elongated QDs.

\section{Conclusion}

We have shown how a more than twofold enhancement of both chip gain and saturation gain of a QD-SOAs can be realized when using elongated QDs, while retaining or even improving ultra-fast gain recovery dynamics. Strikingly, a horizontal aspect ratio of 2 already leads to a gain enhancement of $+2.6 \mathrm{~dB}$ representing $70 \%$ of the overall observed enhancement.

Furthermore, polarization insensitive signal amplification is realized for all elongations through stacking of QDs, forming a vertically coupled confinement region with adjustable emission directionality. As such, our approach makes QD-SOAs more competitive with quantum well-based devices, in particular for applications requiring ultra-fast modulation speeds.

\section{Author Information}

\section{Corresponding Author}

* lath@physik.tu-berlin.de

\section{ORCID}

Ludwig A. Th. Greif:

0000-0002-6732-7062

Alexander Mittelstädt: $\quad 0000-0002-7587-9385$

Stefan T. Jagsch: $\quad$ 0000-0002-2365-0554

Andrei Schliwa:

0000-0001-7085-3680

\section{Notes}

The authors declare no competing financial interest. 


\section{Acknowledgement}

The authors thank the Deutsche Forschungsgemeinschaft (DFG) which founded this work in the frame of the CRC 787.

\section{Supporting Information Available:}

Figures of the capture $(\mathrm{W} \rightarrow 2)$, escape $(2 \rightarrow \mathrm{W})$, excitation $(1 \rightarrow 2)$ and relaxation $(2 \rightarrow 1)$ lifetimes as functions of the optical output power for differently elongated QDs.

\section{References}

(1) Arakawa, Y.; Sakaki, H. Multidimensional Quantum Well Laser and Temperature Dependence of its Threshold Current. Appl Phys Lett 1982, 40, 939-941.

(2) Asada, M.; Miyamoto, Y.; Suematsu, Y. Gain and the Threshold of ThreeDimensional Quantum-Box Lasers. IEEE J Quant Electron 1986, 22, 1915-1921.

(3) Akiyama, T.; Kuwatsuka, H.; Simoyama, T.; Nakata, Y.; Mukai, K.; Sugawara, M.; Wada, O.; Ishikawa, H. Nonlinear gain dynamics in quantum-dot optical amplifiers and its application to optical communication devices. IEEE J Quant Electron 2001, 3\%, 1059-1065.

(4) Sugawara, M.; Akiyama, T.; Hatori, N.; Nakata, Y.; Ebe, H.; Ishikawa, H. Quantum-dot Semiconductor Optical Amplifiers for High-Bit-Rate Signal Processing up to $160 \mathrm{~Gb} / \mathrm{s}$ and a New Scheme of 3 R Regenerators. Meas Sci Technol 2002, 13, 1683-1691.

(5) Akiyama, T.; Sugawara, M.; Arakawa, Y. Quantum-Dot Semiconductor Optical Amplifiers. Proc IEEE 2007, 95, $1757-1766$.

(6) Ben Ezra, Y.; Lembrikov, B. I.; Haridim, M. Ultrafast All-Optical Processor Based on Quantum-Dot Semiconductor Optical Amplifiers. IEEE J Quant Electron 2009, 45, 34-41.
(7) Nurmohammadi, T.; Abbasian, K.; As'Adi, M. J.; Jafari, D. Design of an Ultrafast All-Optical NOR Lógic Gate Based on Mach-Zehnder Interferometer Using Quantum-Dot SOA. Optik - Int J Light Electron Opt 2014, 125, 4023-4029.

(8) Matsumoto, A.; Akahane, K.; Sakamoto, T.; Umezawa, T.; Kanno, A.; Yamamoto, N. Dynamic Characteristics of 20-Layer Stacked QD-SOA with Strain Compensation Technique by Ultrafast Signals using Optical Frequency Comb. Phys Status Solidi A 2017, 214.

(9) Akiyama, T.; Ekawa, M.; Sugawara, M.; Kawaguchi, K.; Sudo, H.; Kuramata, A.; Ebe, H.; Arakawa, Y. An Ultra-Band Semiconductor Optical Amplifier Having an Extremely High Penalty-Free Output Power/of $23 \mathrm{dBm}$ Achieved with Quantum Dots. IEEE Photonic Tech L 2005, 1\%, 1614-1616.

(10) Matsumoto, A.; Takei, Y.; Matsushita, A.; Akahane, K.; Matsushima, Y.; Ishikawa, H.; Utaka, K. Gain Characteristics and Femto-Second Optical Pulse Response of $1550 \mathrm{~nm}$-Band Multi-Stacked QD-SOA Grown on $\operatorname{InP}(311) B$ Substrate. Opt Commun 2015, 344, 51-54.

(11) Akiyama, T.; Kuwatsuka, H.; Hatori, N.; Nakata, Y.; Ebe, H.; Sugawara, M. Symmetric Highly Efficient $(\approx 0 \mathrm{~dB})$ Wavelength Conversion Based on Four-Wave Mixing in Quantum Dot Optical Amplifiers. IEEE Photonic Tech L 2002, 14, 1139-1141.

(12) Kita, T.; Wada, O.; Ebe, H.; Nakata, Y.; Sugawara, M. Polarization-Independent Photoluminescence from Columnar InAs/GaAs Self-Assembled Quantum Dots. Jpn J Appl Phys 2002, 41, 1143-1145.

(13) Yu, P. et al. Optical Anisotropy in Vertically Coupled Quantum Dots. Phys Rev B 1999, 60, 680-685. 
(14) Kita, T.; Jayavel, P.; Wada, O.; Ebe, H.; Nakata, Y.; Sugawara, M. Polarization Controlled Edge Emission from Columnar InAs/GaAs Self-Assembled Quantum Dots. Phys Status Solidi (C) 2003, 1140, 1137-1140.

(15) Kita, T.; Tamura, N.; Wada, O.; Sugawara, M.; Nakata, Y.; Ebe, H.; Arakawa, Y. Artificial Control of Optical Gain Polarization by Stacking Quantum Dot Layers. Appl Phys Lett 2006, 88, 211106.

(16) Usman, M. In-Plane Polarization Anisotropy of Ground State Optical Intensity in InAs/GaAs Quantum Dots. $J$ Appl Phys 2011, 110, 94512.

(17) Usman, M.; Inoue, T.; Harda, Y.; Klimeck, G.; Kita, T. Experimental and Atomistic Theoretical Study of Degree of Polarization from Multilayer InAs/GaAs Quantum Dot Stacks. Phys Rev B 2011. 84, 115321 .

(18) Usman, M.; Tan, Y. H. M.; Ryu, H.; Ahmed, S. S.; Krenner, H. J.; Boykin, T. B.; Klimeck, G. Quantitative Excited State Spectroscopy of a Single InGaAs Quantum Dot Molecule through Multi-Million-Atom Electronic Structure Calculations. Nanotech 2011, 22, 315709 .

(19) Tillmann, K.; Gerthsen, D.; Pfundstein, P.; Förster, A.; Urban, K. Structural transformations and strain relaxation mechanisms of In0.6Ga0.4As islands grown by molecular, beam epitaxy on $\operatorname{GaAs}(001)$ substrates. J Appl Physic 1995, 78, 3824-3832.

(20) Ma, W.; Nötzel, R.; Schönherr, H. P.; Ploog, K. H. Shape transition of coherent three-dimensional (In,Ga)As islands on GaAs(100). Appl Physic Lett 2001, 79, 4219-4221.

(21) Mano, T.; Nötzel, R.; Hamhuis, G. J.; Eijkemans, T. J.; Wolter, J. H. Effect of annealing on formation of self-assembled (In,Ga)As quantum wires on GaAs (100) by molecular beam epitaxy. $J$ Appl Physic 2002, 92, 4043-4046.

(22) Hospodková, A.; Křápek, V.; Mates, T.; Kuldová, K.; Pangrác, J.; Hulicius, E.; Oswald, J.; Melichar, K.; Humličck, J.; Šimeček, T. Lateral shape of/InAs/GaAs quantum dots in vertically correlated structures. J Cryst Growth 2007, 298, 570-573.

(23) Wang, R. H.; Stintz, A.; Varangis, P. M.; Newell, T. C.; Li, H.; Malloy, K. J.; Lester, L. F. Room-temperature operation of InAs quantum-dash lasers on InP [001]. IEEE Photonic Technol Lett 2001, 13, 767-769.

(24) Ukhanov, A. A.; Wang, R. H.; Rotter, T. J.; Stintz, A.; Lester, L. F.; Eliseev, P. G.; Malloy, K. J. Orientation dependence of the optical properties in InAs quantum-dash lasers on InP. Appl Physic Lett 2002, 81, 981-983.

(25) Lelarge, F. et al. Recent Advances on InAs/InP Quantum Dash Based Semiconductor Lasers and Optical Amplifiers Operating at $1.55 \mu \mathrm{m}$. IEEE J Sel Top Quantum Electron 2007, 13, 111-124.

(26) Kayanuma, Y. Quantum-size Effects of Interacting Electrons and Holes in Semiconductor Microcrystals with Spherical Shape. Phys. Rev. B 1988, 38, 9797-9805.

(27) Zhou, D.; Piron, R.; Dontabactouny, M.; Homeyer, E.; Dehaese, O.; Batte, T.; Gicquel, M.; Grillot, F.; Tavernier, K.; Even, J.; Loualiche, S. Effect of stack number on the threshold current density and emission wavelength in quantum dash/dot lasers. Phys Status Solidi Curr Top Solid State Phys 2009, 6, 2217-2221.

(28) Hein, S.; Podemski, P.; Sȩk, G.; Misiewicz, J.; Ridha, P.; Fiore, A.; Patriarche, G.; Höfling, S.; Forchel, A. Orientation dependent emission properties of 
columnar quantum dash laser structures. Appl Phys Lett 2009, 94, 241113(1-3).

(29) Bakonyi, Z.; Su, H.; Onishchukov, G.; Lester, L. F.; Member, S.; Gray, A. L.; Newell, T. C. High-Gain Quantum-Dot Semiconductor Optical Amplifier for 1300 nm. IEEE J Quant Electron 2003, 39, 1409-1414.

(30) Borri, P.; Schneider, S.; Langbein, W.; Bimberg, D. Ultrafast Carrier Dynamics in InGaAs Quantum Dot Materials and Devices. J Optic A 2006, 8.

(31) Jayavel, P.; Kita, T.; Wada, O.; Ebe, H.; Sugawara, M.; Arakawa, Y.; Nakata, Y.; Akiyama, T. Optical Polarization Properties of InAs/GaAs Quantum Dot Semiconductor Optical Amplifier. Jpn J Appl Physic 2005, 44, 2528-2530.

(32) Saito, H.; Nishi, K.; Sugou, S.; Sugimoto, Y. Controlling polarization of quantum-dot surface-emitting lasers by using structurally anisotropic selfassembled quantum dots. Appl Physic Lett 1997, 71, 590.

(33) Usman, M. Atomistic Theoretical Study of Electronic and Polarization Properties of Single and Vertically Stacked Elliptical InAs Quantum Dots. Phys Rev B 2012, 86,155444 .

(34) Ben-Ezra, Y.; Haridim, M.; Lembrikov, B. I. Theoretical Analysis of Gain-Recovery Time and Chirp in QDSOA. IEEE Photonic Tech L 2005, 17, 1803-1805.

(35) Ben-Ezra, Y.; Lembrikov, B. I.; Haridim, M. Acceleration of Gain Recovery and Dynamics of Electrons in QD-SOA. IEEE J Quant Electron 2005, 41, $1268+1273$.

(36) Li, X.; Li, G. Comments on "Theoretical Analysis of Gain-Recovery Time and Chirp in QD-SOA". IEEE Photonic Tech L 2006, 18, 2434-2435.
(37) Qasaimeh, O. Characteristics of CrossGain (XG) Wavelength Conversion in Quantum Dot Semiconductor Optical Amplifiers. IEEE Photonic Tech L 2004, $16,542-544$.

(38) Stier, O.; Grundmann, M.; Bimberg, D. Electronic and Optical Properties of Strained Quantum Dots Modeled by 8Band kp Theory. Phys Rev B 1999, 59, 5688-5701.

(39) Schliwa, A.; Winkelnkemper, M.; Bimberg, D. Impact of Size, Shape, and Composition on Piezoelectric Effects and Electronic Properties of $\operatorname{In}(\mathrm{Ga}) \mathrm{As} / \mathrm{GaAs}$ Quantum Dots. Phys Rev B 2007, 76, 205324.

(40) Greif, L. A.; Jagsch, S. T.; Wagner, M. R.; Schliwa, A. Tuning the Emission Directionality of Stacked Quantum Dots. ACS Photonics 2018, 5, 4838-4845.

(41) Zhao, Y.; Han, W.; Song, J.; Li, X.; Liu, Y.; Gao, D.; Du, G.; Cao, H.; Chang, R. P. H. Spontaneous Emission Factor for Semiconductor Superluminescent Diodes. J Appl Physic 1999, 85, 3945-3948.

(42) Berg, T. W.; Bischoff, S.; Magnusdottir, I.; Moørk, J. Ultrafast Gain Recovery and Modulation Limitations in SelfAssembled Quantum-Dot Devices. IEEE Photonic Tech L 2001, 13, 541-543.

(43) Uskov, A. V.; Berg, T. W.; Mørk, J. Theory of Pulse-Train Amplification Without Patterning Effects in Quantum-Dot Semiconductor Optical Amplifiers. IEEE J Quant Electron 2004, 40, 306-320.

(44) Marcuse, D. Computer Model of an Injection Laser Amplifier. IEEE J Quant Electron 1983, QE-19, 63-73.

(45) Berg, T. W.; Mørk, J. Saturation and noise properties of quantum-dot optical amplifiers. IEEE J Quant Electron 2004, 40, 1527-1539. 